\title{
Spline Interpolation at Knot Averages on a Two-Sided Geometric Mesh*
}

\author{
By M. J. Marsden
}

\begin{abstract}
For splines of degree $k>1$ with knots $-t_{i}=t_{2 m+1-i}=1+q+q^{2}$ $+\cdots+q^{m-i}, i=1, \ldots, m$, where $0<q<1$, it is shown that spline interpolation to continuous functions at nodes $\tau_{i}=\Sigma_{1}^{k} w_{j} t_{i+j}, i=1, \ldots, n=2 m-k-1$, has operator norm $\|P\|$ which is bounded independently of $q$ and $m$ as $q$ tends to zero if and only if $\left(1-w_{1}\right)^{k}<\frac{1}{2},\left(1-w_{k}\right)^{k}<\frac{1}{2}$, and $w_{j}>0, j=1, \ldots, k$. The choice of nodes $\tau_{i}=$ $\Sigma_{0}^{k+1} w_{j} t_{i+j}$ and the growth rate of $\|P\|$ with $k$ are also discussed.
\end{abstract}

1. Two-Sided $q$-Splines. To integers $n>0, k \geqslant 0$, and a nondecreasing sequence $\mathbf{t}=\left(t_{i}\right)_{1}^{n+k+1}$ with $t_{i}<t_{i+k+1}, i=1, \ldots, n$, is associated $\delta_{k+1, t}$, the space of polynomial splines of order $k+1$ with knot sequence $\mathbf{t}$, defined by $\delta_{k+1, t}=$ $\operatorname{span}\left\{N_{1}, \ldots, N_{n}\right\}$, where each $N_{i}=N_{i, k+1}$ is an appropriate normalized $B$-spline. See [1] for specific details.

With $q>0, m$ a positive integer, $n=2 m-k-1$, and

$$
\begin{aligned}
t_{i} & =-\left(1+q+\cdots+q^{m-i}\right), & & i=1, \ldots, m, \\
& =1+q+\cdots+q^{i-m-1}, & i & =m+1, \ldots, 2 m,
\end{aligned}
$$

$\mathfrak{S}_{k+1, \mathrm{t}}$ is the space of two-sided q-splines.

Each two-sided $q$-spline can be represented as

$$
\begin{aligned}
s(t)= & \sum_{1}^{m-1} A_{j}\left[q^{j-m}\left(t_{j+1}-t\right)_{+}\right]^{k}+\sum_{0}^{k} A_{m+j} t^{j} \\
& +\sum_{1}^{m-1} A_{m+k+j}\left[q^{-j}\left(t-t_{m+j}\right)_{+}\right]^{k}
\end{aligned}
$$

where $u_{+}=\max \{u, 0\}$, with the endpoint conditions

$$
s^{(i)}\left(t_{1}\right)=s^{(i)}\left(t_{2 m}\right)=0, \quad i=0, \ldots, k-1 .
$$

Conversely, each function of the form (1.2) which satisfies (1.3) is a two-sided $q$-spline.

With the notation

$$
[i]=1+q+\cdots+q^{i-1}, \quad i=0,1, \ldots,
$$

relations such as

$$
t_{j+1}-t_{i}=q^{m-j}[j+1-i], \quad 0<i \leqslant j<m,
$$

Received May 27, 1980; revised May 7, 1981.

1980 Mathematics Subject Classification. Primary 41A05, 41 A15.

* Research supported by National Research Council of Canada grants A7687 and A7549 while at the University of Alberta on leave from the University of Pittsburgh.

(C) 1982 American Mathematical Society 0025-5718/82/0000-0473/\$04.50 
and

$$
t_{i+1}-t_{j}=q^{j-m}[i+1-j], \quad m<j \leqslant i<2 m,
$$

can be stated in a compact form. The notation

$$
[i] !=[i][i-1] \cdots[2][1] \text { and }\left[\begin{array}{l}
j \\
i
\end{array}\right]=\frac{[j] !}{[i] ![j-i] !}
$$

will also be useful.

The clause "as $q$ tends to zero" appears throughout this paper. It will always mean "for all $q$ satisfying $0<q \leqslant q_{0}$ ". The specific choice of $q_{0}$ will vary from instance to instance. However, $q_{0}$ will never depend on $m$.

LEMMA 1.1. With $k$ and $m$ fixed, let $\{s\}$ be a set of two-sided q-splines with $\left\{\left(A_{1}, \ldots, A_{2 m+k-1}\right)\right\}$ the corresponding set of coefficient vectors in (1.2). Then $\{s\}$ is uniformly bounded as $q$ tends to zero if and only if $\left\{\left(A_{j}\right)\right\}$ is uniformly bounded as $q$ tends to zero. Moreover, if the bound on $\{s\}$ is independent of $m$, then so is the bound on $\left\{\left(A_{j}\right)\right\}$.

Proof. Let $1>q_{0}>0$ and $C$ be such that

$$
\left|A_{j}\right| \leqslant C, \quad \text { all } j \text { and } 0<q \leqslant q_{0} .
$$

Then, for each real $t$ and $0<q \leqslant q_{0}$,

$$
\begin{aligned}
|s(t)| & \leqslant C\left(\sum_{1}^{m-1}\left[q^{j-m}\left(t_{j+1}-t_{1}\right)\right]^{k}+\sum_{0}^{k} t_{2 m}^{j}+\sum_{1}^{m-1}\left[q^{-j}\left(t_{2 m}-t_{m+j}\right)\right]^{k}\right) \\
& =C\left(\sum_{1}^{m-1}[j]^{k}+\sum_{0}^{k}[m]^{j}+\sum_{1}^{m-1}[m-j]^{k} \leqslant(2 m+k-1) C[m]^{k}\right) \\
& <(2 m+k-1) C m^{k} .
\end{aligned}
$$

Conversely, let $1>q_{0}>0$ and $B$ be such that

$$
|s(t)| \leqslant B, \quad \text { all real } t \text { and } 0<q \leqslant q_{0} .
$$

Since

$$
\sum_{0}^{k} A_{m+j}(i / k)^{j}=s(i / k), \quad i=0, \ldots, k,
$$

is a matrix equation with nonsingular coefficient matrix $V=\left((i / k)^{j}\right)$ depending only on $k$,

$$
\left|A_{m+j}\right| \leqslant(k+1) B_{k} B, \quad j=0, \ldots, k,
$$

where $B_{k}$ is a bound on the entries of $V^{-1}$. Set $C_{0}=(k+1) B_{k} B$ and assume inductively that $q_{1}$ is such that $\left|A_{m-j}\right| \leqslant C_{j}$ for $j=0,1, \ldots, i-1$ for $q<q_{1}$. From (1.2)

$$
\begin{aligned}
s\left(t_{m-i}\right)-s\left(t_{m-i+1}\right)= & A_{m-i}+\sum_{1}^{i-1} A_{m-j}\left([i-j+1]^{k}-[i-j]^{k}\right) \\
& +\sum_{1}^{k} A_{m+j}(-1)^{j}\left([i+1]^{j}-[i]^{j}\right),
\end{aligned}
$$


so that

$$
\begin{aligned}
\left|A_{m-i}\right| & \leqslant 2 B+\sum_{1}^{i-1} C_{j}\left([i-j+1]^{k}-[i-j]^{k}\right)+C_{0} \sum_{1}^{k}\left([i+1]^{j}-[i]^{j}\right) \\
& \leqslant 2 B+\sum_{1}^{i-1} C_{j} q^{i-j} k\left(1-q_{0}\right)^{-k}+C_{0} \sum_{1}^{k} q^{i} j\left(1-q_{0}\right)^{-j} \\
& \leqslant 2 B+\sum_{0}^{i-1} C_{j} q^{i-j} R_{k} \quad \text { with } R_{k}=k^{2}\left(1-q_{0}\right)^{-k}
\end{aligned}
$$

Setting $C_{i}=2 B+\Sigma_{0}^{i-1} C_{j} q_{1}^{i-j} R_{k}$ allows the induction to proceed. Then $C_{1}=2 B$ $+C_{0} q_{1} R_{k}$, and $C_{i+1}=q_{1}\left(1+R_{k}\right) C_{i}+2 B\left(1-q_{1}\right), i=1, \ldots, m-2$. This recurrence solves as

$$
\begin{aligned}
C_{i}=\frac{2 B\left(1-q_{1}\right)}{1-q_{1}-q_{1} R_{k}}\left[1-\left(q_{1}+q_{1} R_{k}\right)^{i-1}\right]+C_{1}\left(q_{1}+q_{1} R_{k}\right)^{i-1}, & \\
& i=1, \ldots, m-1,
\end{aligned}
$$

if $q_{1}+q_{1} R_{k} \neq 1$. Imposing the added restriction $q_{1}+q_{1} R_{k}<\frac{1}{2}$ and noting that a symmetric argument will yield $\left|A_{m+k+j}\right| \leqslant C_{j}, j=1, \ldots, m-1$, establishes that

$$
\max _{j}\left|A_{j}\right| \leqslant \max _{i} C_{i} \leqslant 4 B+C_{1}+C_{0} \text {. }
$$

This bound is independent of $m$ if $B$ is independent of $m$.

LEMMA 1.2. Let $k$ and $m$ be fixed. As $q$ tends to zero, the coefficients $\left(A_{j}\right)$ satisfy

$$
A_{i}+\sum_{i+1}^{m-1} A_{j} q^{(j-i)(k-i)}\left[\begin{array}{c}
j \\
i
\end{array}\right]+\sum_{k-i}^{k} A_{m+j} O\left(q^{(m-i)(k-i)}\right)=0, \quad i=1, \ldots, k-1
$$

and

$$
A_{k}+\sum_{k+1}^{m-1} A_{j}\left[\begin{array}{l}
j \\
k
\end{array}\right]+\sum_{0}^{k} A_{m+j}\left(\frac{t_{1}^{j}}{[k] !}+O\left(q^{m-k+1}\right)\right)=0
$$

Proof. This follows from (1.3). Let functionals $\Lambda_{i p}, 1 \leqslant i \leqslant \nu \leqslant k$, be defined by

$$
\Lambda_{1 \nu} s=q^{(m-1)(k-\nu)} \frac{\nu !}{k !}(-1)^{k-\nu} s^{(k-\nu)}\left(t_{1}\right)
$$

and, recursively,

$$
\Lambda_{i \nu} s=q^{\nu-k}\left(\Lambda_{i-1, \nu} s-[i-1] \Lambda_{i-1, \nu-1} s\right) /[i]
$$

From (1.2)

$$
\begin{aligned}
s^{(k-\nu)}\left(t_{1}\right)= & \sum_{1}^{m-1} A_{j} q^{(j-m)(k-\nu)} \frac{k !}{\nu !}(-1)^{k-\nu}[j]^{\nu} \\
& +\sum_{k=\nu}^{k} A_{m+j} \frac{j !}{(j-k+\nu) !} t_{1}^{j-k+\nu}
\end{aligned}
$$

whence

$$
\Lambda_{1 \nu} s=\sum_{1}^{m-1} A_{j} q^{(j-1)(k-\nu)}[j]^{\nu}+\sum_{k-\nu}^{k} A_{m+j} q^{(m-1)(k-\nu)} C_{1 j \nu}
$$


where

$$
C_{1 j \nu}=\frac{\nu ! j !}{k !(j-k+\nu) !}(-1)^{k-\nu} t_{1}^{j-k+\nu} .
$$

The recursion formula gives

$$
\Lambda_{i \nu} s=\sum_{i}^{m-1} A_{j} q^{(j-i)(k-\nu)}[j]^{\nu-i}\left[\begin{array}{l}
j \\
i
\end{array}\right]+\sum_{k=\nu}^{k} A_{m+j} q^{(m-i)(k-\nu)} C_{i j \nu}
$$

where

$$
C_{i j \nu}=\left(C_{i-1, j, \nu}-[i-1] q^{m-i+1} C_{i-1, j, \nu-1}\right) /[i] .
$$

From (1.3) each $\Lambda_{i \nu} s=0$ and, in particular, $\Lambda_{i i} s=0$. This fact, along with the observation that $C_{k j k}=C_{1 j k} /[k] !+O\left(q^{m-k+1}\right)$ completes the proof.

Combining Lemmas 1.1, 1.2, and a symmetric counterpart of Lemma 1.2 yields

LEMMA 1.3. Let $k$ and $m$ be fixed and let $\{s\}$ be a set of two-sided $q$-splines which is bounded as $q$ tends to zero. Then the corresponding set of coefficient vectors $\left\{\left(A_{j}\right)\right\}$ satisfies

$$
\begin{aligned}
A_{i} & =O\left(q^{k-i}\right), & & i=1, \ldots, k-1, \\
A_{i} & =O(1), & i & =k, \ldots, 2 m, \\
A_{2 m+i} & =O\left(q^{i}\right), & i & =1, \ldots, k-1,
\end{aligned}
$$

as $q$ tends to zero. If the bound on $\{s\}$ is independent of $m$, then so are the bounds on the $A_{j}$.

The independence of $m$ in the $O\left(q^{k-i}\right)$ and $O\left(q^{i}\right)$ bounds follows from the exponential decay of the coefficients in the first $k-1$ equations of Lemma 1.2.

2. Spline Interpolation. Let $\tau=\left(\tau_{i}\right)_{1}^{n}$ be a strictly increasing sequence. It is known [1] that: For each function $f$ defined on $\tau$ there is exactly one $s \in \mathcal{S}_{k+1, t}$ such that $s\left(\tau_{i}\right)=f\left(\tau_{i}\right), i=1, \ldots, n$, if and only if $N_{i}\left(\tau_{i}\right)>0, i=1, \ldots, n$, or, equivalently, if and only if

$$
t_{i}<\tau_{i}<t_{i+k+1}, \quad i=1, \ldots, n .
$$

When $\tau$ satisfies (2.1) a linear map $P$ into $\varsigma_{k+1, t}$ which reproduces $\delta_{k+1, t}$ may be defined by: For each function $f$ defined on $\tau, P f \in \mathcal{S}_{k+1, t}$ and $(P f)\left(\tau_{i}\right)=f\left(\tau_{i}\right)$, $i=1, \ldots, n$. In fact, $P f=\sum f\left(\tau_{j}\right) L_{j}$ where $\left(L_{j}\right)_{1}^{n}$ is defined by $L_{j}\left(\tau_{i}\right)=\delta_{i j}, i, j=$ $1, \ldots, n$. The operator norm of $P$ is

$$
\|P\|=\sup _{f} \frac{\|P f\|}{\|f\|}
$$

where the sup is taken over all $f \in C\left[t_{1}, t_{n+k+1}\right]$ and

$$
\|f\|=\sup \left\{|f(t)|: t_{1} \leqslant t \leqslant t_{n+k+1}\right\} .
$$

It is well known that

$$
\|P\|=\max _{t} \sum_{1}^{n}\left|L_{j}(t)\right|=\max _{0<\mu<n}\left(\max _{\tau_{\mu}<t<\tau_{\mu+1}} s_{\mu}(t)\right),
$$


where $\tau_{0}=t_{1}, \tau_{n+1}=t_{n+k+1}$ and $\left(s_{\mu}\right)_{0}^{n}$ is defined by

$$
\begin{aligned}
s_{\mu}\left(\tau_{i}\right) & =(-1)^{i+\mu}, & & i=1, \ldots, \mu, \\
& =-(-1)^{i+\mu}, & & i=\mu+1, \ldots, n .
\end{aligned}
$$

For each $\mu$, the so-called Lebesgue function $\Sigma\left|L_{i}(t)\right|$ coincides with $s_{\mu}(t)$ on the interval $\left[\tau_{\mu}, \tau_{\mu+1}\right]$.

One way of specifying $\tau$ is to require that the nodes be knot averages, i.e.,

$$
\tau_{i}=\sum_{0}^{k+1} w_{j} t_{i+j}, \quad i=1, \ldots, n,
$$

where the $w_{j}$ are fixed nonnegative numbers which sum to one.

TheOREM 1. Let $k \geqslant 2, m$, and $\left(w_{i}\right)_{0}^{k+1}$ be fixed. Let $\mathbf{t}$ be given by (1.1) and $\tau$ be given by (2.3). If $\|P\|$ is bounded as $q$ tends to zero, then

$$
w_{i}>0, \quad i=1, \ldots, k .
$$

If the bound on $\|P\|$ is also independent of $m$, then either

$$
w_{0}=0 \text { and }\left(1-w_{1}\right)^{k}<\frac{1}{2}
$$

or

$$
w_{0}>0 \text { and } \frac{1}{2}<\left(1-w_{0}\right)^{k}
$$

and, either

$$
w_{k+1}=0 \text { and }\left(1-w_{k}\right)^{k}<\frac{1}{2}
$$

or

$$
w_{k+1}>0 \text { and } \frac{1}{2}<\left(1-w_{k+1}\right)^{k} \text {. }
$$

Conversely, if (2.4), (2.5), (2.6) hold, then $\|P\|$ is bounded independently of $m$ as $q$ tends to zero.

Proof. Let $w_{a}$ be the first positive weight and $w_{b}$ be the last positive weight, so that $\tau_{i}=\sum_{a}^{b} w_{j} t_{i+j}$, and set

$$
\begin{aligned}
& \theta_{1}=\left(1-w_{a}\right)+\left(1-w_{a}-w_{a+1}\right) q+\cdots+w_{b} q^{b-a-1} \\
& \theta_{2}=\left(1-w_{b}\right)+\left(1-w_{b}-w_{b-1}\right) q+\cdots+w_{a} q^{b-a-1}
\end{aligned}
$$

If $a=b$, then $\theta_{1}=\theta_{2}=0$. If $a<b$, then $0<\theta_{1}<1$ and $0<\theta_{2}<1$ as $q$ tends to zero. Therefore,

$$
\begin{aligned}
t_{i+b-1} & <\tau_{i}=t_{i+b}-\theta_{2} q^{m+1-b-i} \leqslant t_{i+b}, & & i=1, \ldots, m-b, \\
t_{i+a} \leqslant \tau_{i} & =t_{i+a}+\theta_{1} q^{i+a-m}<t_{i+a+1}, & & i=m-a+1, \ldots, n,
\end{aligned}
$$

for all sufficiently small $q>0$. Since

$$
\tau_{i}=1-2 \sum_{a}^{m-i} w_{j}+O(q), \quad i=m-b+1, \ldots, m-a
$$

as $q$ tends to zero, it follows that also

$$
-1<\tau_{m-b+1}<\tau_{m-b+2}<\cdots<\tau_{m-a}<+1
$$

for all sufficiently small $q>0$. 
Henceforth, we require that $q$ be such that the inequalities in (2.7) and (2.9) hold. This requirement is independent of $m$.

Now let $\|P\|$ be bounded independently of $m$ as $q$ tends to zero. We shall prove that (2.4) and (2.6) must hold. A symmetric argument, which we omit, will give (2.5).

Let $s=s_{\mu}$ be defined by (2.2) with $\mu<m-b+1$ or $\mu>m-a-1$. There is a constant $C$ which bounds $\|P\|$ so that $\|s\| \leqslant C$ as $q$ tends to zero. Since the restriction of $s$ to $[-1,+1]$ is a polynomial of degree $k$, it follows from a theorem of A. A. Markov (see [7]) that

$$
\max \left\{\left|s^{\prime}(t)\right|:-1 \leqslant t \leqslant 1\right\} \leqslant C k^{2} .
$$

Thus, (2.8), (2.9), and the mean-value theorem imply that

$$
2=\left|s\left(\tau_{i}\right)-s\left(\tau_{i+1}\right)\right| \leqslant C k^{2}\left(\tau_{i+1}-\tau_{t}\right) \leqslant 2 C k^{2} w_{m-i}+O(q)
$$

for $i=m-b+1, \ldots, m-a-1$ as $q$ tends to zero. Thus, $w_{i} \geqslant 1 / C k^{2}>0$, $i=a+1, \ldots, b-1$.

Suppose that $b<k$. Then, on the one hand, (1.2) gives

$$
\begin{aligned}
\pm 1= & s\left(\tau_{1}\right)=\sum_{b}^{m-1} A_{j}\left([j-b]+\theta_{2} q^{j-b}\right)^{k}+\sum_{0}^{k} A_{m+j}\left(-[m-b]-\theta_{2} q^{m-b}\right)^{j} \\
= & A_{b} \theta_{2}^{k}+\sum_{b+1}^{m-1} A_{j}\left([j-b]^{k}+O\left(q^{j-b}\right)\right) \\
& +\sum_{0}^{k} A_{m+j}\left((-[m-b])^{j}+O\left(q^{m-b}\right)\right)
\end{aligned}
$$

whereas, on the other hand, with $\Lambda_{i i} s$ as in the proof of Lemma 1.2,

$$
\begin{aligned}
0= & \theta_{2}^{k} \Lambda_{b b} s+\sum_{b+1}^{k-1}[i-b]^{k} \Lambda_{i i} s+[k] ! \Lambda_{k k} s \\
= & A_{b} \theta_{2}^{k}+\sum_{b+1}^{m-1} A_{j}\left([j-b]^{k}+O\left(q^{j-b}\right)\right) \\
& +\sum_{0}^{k} A_{m+j}\left((-[m-b])^{j}+O\left(q^{m-b}\right)\right) .
\end{aligned}
$$

Subtraction yields

$$
\pm 1=\sum_{b+1}^{m-1} A_{j} O\left(q^{j-b}\right)+\sum_{0}^{k} A_{m+j} O\left(q^{m-b}\right),
$$

so that $\left(A_{j}\right)$ cannot be bounded as $q$ tends to zero. This contradiction to Lemma 1.3 shows that $b \geqslant k$.

A similar argument with $s\left(\tau_{n}\right)$ shows that $a \leqslant 1$, so that (2.4) is proved.

To prove (2.6), we first suppose that $w_{k+1}=0$. We must show that $\left(1-w_{k}\right)^{k}<\frac{1}{2}$ or, equivalently, that

$$
r_{2}=\theta_{2}^{k} /\left(1-\theta_{2}^{k}\right)<1 \text { as } q \text { tends to zero. }
$$

Again, let $s=s_{\mu}$ be defined by (2.2). Then Lemma 1.2 and (1.2) give

$$
-s\left(\tau_{1}\right)=[k] ! \Lambda_{k k} s-s\left(\tau_{1}\right)=\sum_{0}^{m-k-1} M_{0 j} A_{k+j}+\sum_{0}^{k} R_{0 j} A_{m+j}
$$


and

(2.12)

$$
s\left(\tau_{i}\right)-s\left(\tau_{i+1}\right)=\sum_{i=1}^{m-k-1} M_{i j} A_{k+j}+\sum_{0}^{k} R_{i j} A_{m+j}, \quad i=1, \ldots, m-k-1,
$$

where

$$
\begin{aligned}
M_{0 j} & =[k+j] ! /[j] !-\left([j]+\theta_{2} q^{j}\right)^{k}, \quad j=0, \ldots, m-k-1, \\
M_{i, i-1} & =\theta_{2}^{k}, \quad i=1, \ldots, m-k-1, \\
M_{i j} & =\left([j-i+1]+\theta_{2} q^{j-i+1}\right)^{k}-\left([j-i]+\theta_{2} q^{j-i}\right)^{k}, \\
& i=1, \ldots, m-k-1 ; j=i, \ldots, m-k-1, \\
R_{0 j} & =[k] ! C_{k j k}-\tau_{1}^{j}, \quad j=0, \ldots, k, \\
R_{i j} & =\tau_{i}^{j}-\tau_{i+1}^{j}, \quad i=1, \ldots, m-k-1 ; j=0, \ldots, k,
\end{aligned}
$$

with $C_{k j k}=t_{1}^{k} /[k] !+O\left(q^{m-k+1}\right)$ as in the proof of Lemma 1.2.

Since the $A_{j}$ are bounded and

$$
\begin{aligned}
M_{i i} & =1-\theta_{2}^{k}+O(q), \quad i=0, \ldots, m-k-1, \\
M_{0 j} & <[k+j]^{k}-[j]^{k}<q^{j}[k] k[k+j]^{k-1}<q^{j k}(1-q)^{-k}, \\
M_{i j} & <[j-i+2]^{k}-[j-i]^{k}<q^{j-i} k(1-q)^{-k}, \\
& j=i+1, \ldots, m-k-1, \\
\left|R_{0 j}\right| & <q^{m-k}(j+1)(1-q)^{-j}, \\
\left|R_{i j}\right| & \leqslant q^{m-k-i j(1-q)^{-j},}
\end{aligned}
$$

the system (2.11) and (2.12) has the form

$$
\begin{gathered}
\left(1-\theta_{2}^{k}\right) A_{k}=-s\left(\tau_{1}\right)+O(q), \\
\theta_{2}^{k} A_{k+i-1}+\left(1-\theta_{2}^{k}\right) A_{k+i}=s\left(\tau_{i}\right)-s\left(\tau_{i+1}\right)+O(q), \quad i=1, \ldots, m-k-1,
\end{gathered}
$$

which solves as

$$
A_{k+i}=\frac{2(-1)^{i+\mu}}{1-2 \theta_{2}^{k}}\left[1-\frac{r_{2}^{i+1}}{2 \theta_{2}^{k}}\right]+O(q)
$$

$$
i=0, \ldots, \min (\mu-1, m-k-1),
$$

$$
\begin{aligned}
A_{k+\mu+i}=\frac{2(-1)^{i+1}}{1-2 \theta_{2}^{k}}\left[1-\frac{r_{2}^{i+1}}{\theta_{2}^{i}}+\frac{r_{2}^{i+\mu+1}}{2 \theta_{2}^{k}}\right]+O(q), \\
\quad i=0, \ldots, m-k-1-\mu,
\end{aligned}
$$

if $r_{2} \neq 1$ and as

$$
\begin{aligned}
A_{k+1} & =(-1)^{i+1}(2+4 i)+O(q), \quad i=0, \ldots, \min (\mu-1, m-k-1), \\
A_{k+\mu+i} & =(-1)^{i+1}(2+4 i-4 \mu)+O(q), \quad i=0, \ldots, m-k-1-\mu,
\end{aligned}
$$

if $r_{2}=1$ provided that the buildup of $O(q)$ terms is bounded independently of $m$. This will be the case if $q r_{2}<1$ as $q$ tends to zero, a condition that can be met independently of $m$. By Lemma 1.3 these $A_{j}$ are bounded independently of $m$. Therefore, (2.10) must hold and $\left(1-w_{k}\right)^{k}<\frac{1}{2}$. 
To complete the proof of (2.6) we now suppose that $w_{k+1}>0$. Since $\theta_{2}=1-$ $w_{k+1}+O(q)$, we must now show that $\theta_{2}^{k}>\frac{1}{2}$ as $q$ tends to zero, that is

$$
r_{2}=\theta_{2}^{k} /\left(1-\theta_{2}^{k}\right)>1 \text { as } q \text { tends to zero. }
$$

Since the $\tau_{i}$ have "moved over one interval", Eqs. (2.11) and (2.12) are replaced by

$$
\begin{aligned}
-s\left(\tau_{1}\right)= & {[k] ! A_{k}+\sum_{1}^{m-k-1}\left([k+j] ! /[j] !-\left([j-1]+\theta_{2} q^{j-1}\right)^{k}\right) A_{k+j} } \\
& +\sum_{0}^{k} R_{0 j} A_{m+j}
\end{aligned}
$$

and

$$
\begin{aligned}
s\left(\tau_{i}\right)-s\left(\tau_{i+1}\right)=\sum_{i}^{m-k-1} M_{i, j-1} A_{k+j} & +\sum_{0}^{k} R_{i j} A_{m+j}, \\
i & =1, \ldots, m-k-2,
\end{aligned}
$$

and the bounds on $M_{0 j}$ and $R_{i j}$ are replaced by

$$
\begin{gathered}
\left|[k+j] ! /[j] !-\left([j-1]+\theta_{2} q^{j-1}\right)^{k}\right|<q^{j-1} k(1-q)^{-k}, \\
\left|R_{0 j}\right|<q^{m-k-1}(j+1)(1-q)^{-j}, \\
\left|R_{i j}\right| \leqslant q^{m-k-1-i} j(1-q)^{-j}
\end{gathered}
$$

This incomplete system now has the form

$$
\begin{gathered}
A_{k}+\left(1-\theta_{2}^{k}\right) A_{k+1}=-s\left(\tau_{1}\right)+O(q), \\
\theta_{2}^{k} A_{k+i}+\left(1-\theta_{2}^{k}\right) A_{k+i+1}=s\left(\tau_{i}\right)-s\left(\tau_{i+1}\right)+O(q), \quad i=1, \ldots, m-k-2 .
\end{gathered}
$$

Adding the equation

$$
s\left(\tau_{m-k-1}\right)=A_{m-1} \theta_{2}^{k}+\sum_{0}^{k} A_{m+j} \tau_{m-k-1}^{j}=A_{m-1} \theta_{2}^{k}+s(-1)+O(q)
$$

and imposing the restriction $q r_{2}^{-1}<1$ permits us to solve this system backwards in terms of $s(-1)$ as

$$
\begin{aligned}
A_{m-i}= & \frac{(-1)^{m-1-k-\mu-i}}{2 \theta_{2}^{k}-1}\left[2-\left(1+r_{2}\right) r_{2}^{-i}\right]+\left(1+r_{2}\right)\left(-r_{2}\right)^{-i} s(-1) \\
& +O(q), \quad i=1, \ldots, m-k-1-\mu \\
A_{k+\mu+1-i}= & \frac{(-1)^{i-1}}{2 \theta_{2}^{k}-1}\left[2-\left(1+r_{2}\right) r_{2}^{-i}\left(2-r_{2}^{-m+k+\mu+1}\right)\right] \\
& +\left(1+r_{2}\right)\left(-r_{2}\right)^{-m+k+\mu+1-i} s(-1)+O(q), \quad i=1, \ldots, \mu,
\end{aligned}
$$

if $0 \leqslant \mu \leqslant m-k-2$ and as

$$
\begin{aligned}
A_{m-i}= & \frac{(-1)^{\mu-m+k-i}}{2 \theta_{2}^{k}-1}\left[2-\left(1+r_{2}\right) r_{2}^{-i}\right] \\
& +\left(1+r_{2}\right)\left(-r_{2}\right)^{-i} s(-1)+O(q), \quad i=1, \ldots, m-k-1,
\end{aligned}
$$

if $\mu \geqslant m-k-1$. Since the $A_{j}$ are bounded independently of $m,(2.14)$ must hold and $\left(1-w_{k+1}\right)^{k}>\frac{1}{2}$. 
The proof that (2.4), (2.5), (2.6) are necessary conditions for $\|P\|$ to be bounded independently of $m$ as $q$ tends to zero is complete.

To prove that (2.4), (2.5), (2.6) are sufficient that $\|P\|$ be bounded independently of $m$ as $q$ tends to zero, we will use the approach outlined in the proof of Lemma 1.1. That is, we will first show that, for each $s=s_{\mu}$, the block $A_{m}, \ldots, A_{m+k}$ is bounded and then argue recursively from bounds on $s\left(\tau_{i}\right)$ (replacing $s\left(t_{i}\right)$ in the proof of Lemma 1.1) that $A_{m-i}$ (and $A_{m+k+i}$ ), $i=1, \ldots, m-1$, are bounded independently of $m$. Finally, we will use (1.2) and (2.13) or (2.18) or (2.19) to bound $s_{\mu}(t)$ for all $t$ and all $\mu$.

If $a=0$ and $b=k+1$, the first step, bounding the block $A_{m}, \ldots, A_{m+k}$ is easy since (2.9) implies that

$$
\sum_{0}^{k} A_{m+j} \tau_{m-k+i}^{j}= \pm 1, \quad i=k+1-b, \ldots, k-a,
$$

and (2.4), (2.8) give a bounded inverse for the Vandermonde matrix $\left(\tau_{m-k+i}^{j}\right)$. However, if $b=k$ then the $i=0$ equation of (2.20) is replaced by

$$
\theta_{2}^{k} A_{m-1}+\sum_{0}^{k} A_{m+j} \tau_{m-k}^{j}= \pm 1 .
$$

If $a=1$, there is a similar replacement of

$$
\sum_{0}^{k} A_{m+j} \tau_{m}^{j}+\theta_{1}^{k} A_{m+k+1}= \pm 1
$$

for the $i=k$ equation of (2.20).

Therefore, if $b=k$ (and/or $a=1$ ), a preliminary step to eliminate $\theta_{2}^{k} A_{m-1}$ from (2.21), at the expense of adding a bounded quantity to the right member, is necessary. While eliminating $\theta_{2}^{k} A_{m-1}$ through a sequence of upper triangulation steps on (2.11), (2.12), (2.21) is straightforward, there must be an argument that $\theta_{2}^{k} A_{m-1}$ is bounded independently of $m$ as $q$ tends to zero independently of $m$. The following lines supply this argument.

Let $b=k$ and let $s$ be any of the $s_{\mu}$ given by (2.2). Using the bounds on $M=\left(M_{i j}\right)$, we see that this matrix is diagonally dominant if $q$ is such that $1-\theta_{2}^{k}>\theta_{2}^{k}+k q(1-q)^{-k-1}$. But (2.5) $a$ is equivalent to $1-\theta_{2}^{k}>\theta_{2}^{k}$ for sufficiently small $q$, so that this condition can be met by imposing a further restriction on $q$.

Let $q_{0}>0$ and $\delta>0$ be such that $\delta=1-2 \theta_{2}^{k}-k q_{0}\left(1-q_{0}\right)^{-k-1}$. Then the solutions of a system

$$
M \mathbf{x}=\mathbf{b}
$$

satisfy $\max _{i}\left|x_{i}\right| \leqslant \delta^{-1} \max _{i}\left|b_{i}\right|$ by the usual diagonal dominance argument. Applying this fact with

$$
\begin{aligned}
b_{0} & =[k] ! \Lambda_{k k} s-s\left(\tau_{1}\right)=-s\left(\tau_{1}\right), \\
b_{i} & =s\left(\tau_{i}\right)-s\left(\tau_{i+1}\right), \quad i=1, \ldots, m-k-1,
\end{aligned}
$$

as well as with

$$
b_{i}=-R_{i j}, \quad i=0, \ldots, m-k-1
$$


for each $j=0, \ldots, k$, yields

$$
A_{m-1}=C+\sum_{0}^{k} C_{j} A_{m+j}
$$

with

$$
\begin{aligned}
|C| & \leqslant \delta^{-1} \max \left\{\left|s\left(\tau_{1}\right)\right|,\left|s\left(\tau_{i}\right)-s\left(\tau_{i+1}\right)\right|: i=1, \ldots, m-k-1\right\}=2 / \delta \\
\left|C_{0}\right| & <\delta^{-1} \max _{i}\left|R_{i 0}\right|=\left|R_{00}\right| / \delta=O\left(q^{m-k}\right), \\
\left|C_{j}\right| & <\delta^{-1} \max _{i}\left|R_{i j}\right|=\left|\tau_{m-k-1}^{j}-\tau_{m-k}^{j}\right| / \delta \\
& =\left|\left(1+q+\theta_{2} q^{2}\right)^{j}-\left(1+\theta_{2} q\right)^{j}\right| / \delta<q[2] j[3]^{j-1} / \delta \\
& <q j[3]^{j} / \delta=O(q), \quad j=1, \ldots, k .
\end{aligned}
$$

Combining these deductions with (2.21) gives the equation

$$
\sum_{0}^{k} A_{m+j}\left(\tau_{m-k}^{j}+C_{j} \theta_{2}^{k}\right)=s\left(\tau_{m-k}\right)-C \theta_{2}^{k},
$$

which can be adjoined to (2.20). Since $C_{j}=O(q)$ and $\tau_{m-k+1}-\tau_{m-k}=2 w_{k}+$ $O(q)$, the resulting system has a bounded solution as $q$ tends to zero. We have assumed that $a=0$. If $a=1$, a similar argument at $\tau_{m}$ is needed.

We have completed the first step in the proof of sufficiency, i.e., we have shown that the set $A_{m}, \ldots, A_{m+k}$ is bounded. But now (2.12) or (2.16) and their symmetric counterparts imply immediately that the set $A_{k}, \ldots, A_{2 m}$ is bounded. An argument similar to the proof of Lemma 1.2 gives $O\left(q^{i}\right)$ bounds on $A_{k-i}$ and $A_{2 m+i}, i=1, \ldots, k-1$. The second step in the proof is completed.

Now we must bound $s_{\mu}(t)$ for all $t$ and all $\mu$. For $-1 \leqslant t \leqslant+1$, the boundedness of $A_{m}, \ldots, A_{m+k}$ and (2.4) give a uniform bound on $s_{\mu}(t)$. If $t_{1} \leqslant t \leqslant t_{m}$, there is a $\theta_{t}$ in $[0,1]$ and an $i>0$ such that $t_{m-i} \leqslant t=t_{m-i+1}-\theta_{t} q^{i}=-[i]-\theta_{t} q^{i}<$ $t_{m-i+1}$. Then

$$
s(t)=\sum_{m-i}^{m-1} A_{j}\left([i+j-m]+\theta_{t} q^{i+j-m}\right)^{k}+\sum_{0}^{k} A_{m+j} t^{j} .
$$

If $i<m-b$, then $\tau_{m+1-b-i}=-[i]-\theta_{2} q^{i}$ and

$$
|s(t)| \leqslant\left|s\left(\tau_{m+1-b-i}\right)\right|+\left|A_{m-i}\right|+O(q)=1+\left|A_{m-i}\right|+O(q)
$$

can be easily shown. If $i>m-b$, a modified argument gives

$$
|s(t)|<\left|s\left(\tau_{1}\right)\right|+\sum_{1}^{k}\left|A_{j}\right|+O(q)=1+\left|A_{k}\right|+O(q) .
$$

Thus, the $s_{\mu}(t)$ are uniformly bounded for all $\mu$ and all $t$ so that $\|P\|$ is bounded independently of $m$ as $q$ tends to zero.

3. Two Special Cases. Theorem 1 provides counterexamples when (2.4), (2.5), (2.6) are not satisfied, e.g., interpolation at the knots with $k \geqslant 2$ or interpolation at weighted two-knot averages with $k \geqslant 3$. The condition that $q$ tend to zero compares (contrasts?) with the often-used condition that the local mesh ratios $\left(t_{j+1}-t_{j}\right) /\left(t_{i+1}-t_{i}\right),|i-j|=1$ be bounded. 
For $k \geqslant 3$ and $q=1$, it is easy to select weights $w_{j}$ satisfying (2.4), (2.5), (2.6) which still produce unbounded spline interpolation. Thus, even for two-sided $q$-splines, these conditions are not sufficient to guarantee bounded interpolation. Indeed, the method of their derivation suggests that they are linked quite closely to the tendency of $q$ to zero.

For the two special cases which follow it is not clear that we need $q$ to tend to zero. Computational evidence with small $k$ suggests, in fact, that $q$ tending to zero gives "worst-case" results. Thus, Theorems 2 and 3 are imperfect in that the condition that $q$ tend to zero may be superfluous.

THEOREM 2. Let $\mathbf{t}$ be given by(1.1) and, for each $k \geqslant 1$ and $m>k$, let $\tau$ be given by

$$
\tau_{i}=\left(t_{i+1}+t_{i+2}+\cdots+t_{i+k}\right) / k, \quad i=1, \ldots, n .
$$

Then, $\|P\|$ is bounded as $q$ tends to zero. Moreover, there exist absolute constants $1<C_{1}<C_{2}$ such that, for each $k \geqslant 2$,

$$
C_{1}^{k}<\|P\|<C_{2}^{k} \text { as } q \text { tends to zero. }
$$

THEOREM 3. Let $\mathbf{t}$ be given by (1.1) and, for each $k \geqslant 1$ and $m>k$, let $\tau$ be given by (2.3) with

$$
\begin{aligned}
& w_{0}=w_{k+1}=\sin ^{2}\left(\alpha_{k} / 2\right), \\
& w_{j}=\sin \left(\alpha_{k}\right) \sin \left(2 j \alpha_{k}\right), \quad j=1, \ldots, k,
\end{aligned}
$$

where $\alpha_{k}=\pi /(2 k+2)$. Then, $\|P\|$ is bounded as $q$ tends to zero. Moreover, there exist absolute constants $0<C_{3}<C_{4}$ such that, for each $k \geqslant 2$,

$$
C_{3} \log k<\|P\|<C_{4} \log k \text { as } q \text { tends to zero. }
$$

Proof of Theorems 2 and 3. The assertions that $\|P\|$ is bounded as $q$ tends to zero are proved by showing that (2.4), (2.5), (2.6) hold. These follow readily, since, in Theorem 2,

$$
\left(1-w_{k}\right)^{k}=\left(1-w_{1}\right)^{k}=(k-1)^{k} / k^{k}<1 / e<3 / 8
$$

while, in Theorem 3,

$$
\begin{aligned}
\left(1-w_{k+1}\right)^{k} & =\left(1-w_{0}\right)^{k}=\cos ^{2 k}\left(\alpha_{k} / 2\right)>\left(1-\alpha_{k}^{2} / 8\right)^{2 k} \\
& >1-\pi \alpha_{k} / 8>(8 k+3) /(8 k+8)>3 / 4 .
\end{aligned}
$$

In Theorem 2 , the lower bound on $\|P\|$ follows from the fact that, as $q$ tends to zero, the nodes

$$
\tau_{m-k+1}, \tau_{m-k+2}, \ldots, \tau_{m-k+j}, \ldots, \tau_{m-1}
$$

tend to

$$
(2-k) / k,(4-k) / k, \ldots,(2 j-k) / k, \ldots,(k-2) / k,
$$

and that, for $s=s_{\mu}$ with $m-k \leqslant \mu \leqslant m-1$,

$$
|s( \pm 1)|=\left(1-r_{2}^{m-k}\right) /\left(1-2 \theta_{2}^{k}\right)+O(q) \geqslant 1 /\left(1-\theta_{2}^{k}\right)+O(q)>1,
$$

so that $\|P\|$ is bounded below by any lower bound for polynomial interpolation on $[-1,+1]$ at the equally-spaced nodes

$$
-1,(2-k) / k,(4-k) / k, \ldots,(2 j-k) / k, \ldots,(k-2) / k,+1 .
$$


See Rivlin [7, pp. 96-99] for a proof that such polynomial interpolation grows exponentially.

Similarly, in Theorem 3, the lower bound on $\|P\|$ follows from the fact that $\tau_{m-k}, \ldots, \tau_{m}$ approach the Chebyshev nodes $-\cos \left(2 j \alpha_{k}-\alpha_{k}\right), j=1, \ldots, k+1$, as $q$ tends to zero and the fact that polynomial interpolation on these nodes has logarithmic growth. See [7, pp. 93-96].

To complete the proof that $\|P\|$ grows exponentially or logarithmically in Theorem 2 or Theorem 3, respectively, it is necessary only to show that, for each $\mu$, $s_{\mu}(t)$ is "controlled" outside $(-1,+1)$. This fact follows from the closing lines of the proof of Theorem 1 , where it was noted that, for $t_{1} \leqslant t \leqslant t_{m}$, there is a $j<m$ such that $|s(t)| \leqslant 1+\left|A_{j}\right|+O(q)$. For Theorem 2, (3.1) and (2.13) imply that

$$
\max _{j<m}\left|A_{j}\right|<\frac{2}{1-2 \theta_{2}^{k}}+O(q)<\frac{2 e}{e-2}+O(q)<8,
$$

so that $|s(t)|<10$ for $t \leqslant-1$ as $q$ tends to zero. For Theorem 3, (3.2) and (2.18), (2.19) imply that

$$
\begin{aligned}
\max _{j<m}\left|A_{j}\right| & <\frac{2}{2 \theta_{2}^{k}-1}+2|s(-1)|+O(q) \\
& <\frac{2}{2 \cos ^{2 k}\left(\alpha_{k} / 2\right)-1}+2|s(-1)|+O(q) \\
& <4+2|s(-1)|+O(q),
\end{aligned}
$$

so that $|s(t)|<6+2|s(-1)|$ for $t \leqslant-1$ as $q$ tends to zero. Symmetry considerations give like bounds for $|s(t)|$ on $+1=t_{m+1} \leqslant t \leqslant t_{2 m}$.

The proof of Theorem 2 and Theorem 3 is complete.

If $q=1$ (not covered by these theorems), two-sided $q$-spline interpolation is essentially the same as cardinal spline interpolation, for which logarithmic growth of $\|P\|$ with $k$ has been demonstrated; see [6]. This fact supports the conjecture that $q$ tending to zero gives "worst-case" results for the nodes (3.1).

For cubic spline interpolation with arbitrary knot spacing and the nodes (3.1), de Boor [2] has shown that $\|P\|<27$. He conjectures that $\|P\|<3$ or 4 may be true. The following supplies a lower bound on lim sup $\|P\|$, where the lim sup is taken over all ordered knot spacings.

Theorem 4. Let $k=3$ and let $\mathbf{t}$ and $\tau$ be given by (1.1) and (3.1), respectively. Then

$$
\lim \|P\|=(222 \sqrt{111}+999) / 1331=2.507825 \ldots,
$$

where $\lim \|P\|$ denotes the limiting value of $\|P\|$ as $q$ tends to zero and $m$ tends to infinity.

Proof. Let $s=s_{\mu}$ with $\mu=m-1$. From (2.21) and (2.13)

$$
s(-1)=\frac{(-1)^{\mu-m+k}}{1-2 \theta_{2}^{k}}\left(1-r_{2}^{m-k}\right)+O(q)
$$


for each $k \geqslant 2$ and $\mu \geqslant m-k$. Similarly,

$$
s(+1)=\frac{(-1)^{m-1-\mu}}{1-2 \theta_{1}^{k}}\left(1-r_{1}^{m-k}\right)+O(q)
$$

for $k \geqslant 2$ and $\mu \leqslant m-1$. Thus, for the case presently under consideration, $s(t)$ tends, on $[-1,+1]$, to the cubic $p(t)$ satisfying $p( \pm 1)=27 / 11$ and $p( \pm 1 / 3)=$ \pm 1 . This cubic is

$$
p(t)=\left(-297 t^{3}+243 t^{2}+297 t-27\right) / 88 \text {. }
$$

It has a maximum on $[-1,+1]$ of $(222 \sqrt{111}+999) / 1331$ at $t=$ $(9+2 \sqrt{111}) / 33$. Showing that $\lim \|P\|$ exists and is equal to this maximum requires a discussion (which we omit) similar to the last paragraph in the proof of Theorem 1 above.

For arbitrary $k$ it is easy to find $p(t)$, the polynomial which $s_{m-1}(t)$ approaches as $q$ tends to zero and $m$ tends to infinity. From (3.1) and (3.4)

$$
\lim s(+1)=z_{k}=\frac{1}{1-2((k-1) / k)^{k}} .
$$

From (3.3), $\lim s(-1)=(-1)^{k-1} z_{k}$. Then standard combinatorial formulas give (see Gould [4, p. 59])

$$
p(t)=-(-1)^{l} \sum_{0}^{l} \frac{(-4)^{j} T}{T+j}\left(\begin{array}{c}
T+j \\
2 j
\end{array}\right)+\frac{2 T+k z_{k}}{T+l}\left(\begin{array}{c}
T+l \\
2 l
\end{array}\right),
$$

if $k$ is even with $l=k / 2$ and $T=l t$, and

$$
p(t)=-(-1)^{l} \sum_{0}^{l} \frac{(-4)^{j} 2 T}{2 j+1}\left(\begin{array}{c}
T+j-1 / 2 \\
2 j
\end{array}\right)+\frac{2 T+k z_{k}}{k}\left(\begin{array}{c}
T+l-1 / 2 \\
2 l
\end{array}\right),
$$

if $k$ is odd with $l=(k-1) / 2$ and $T=k t / 2$. The maximum of $p(t)$ on $(k-2) / k$ $\leqslant t \leqslant+1$ is a good lower bound on $\|P\|$ as $q$ tends to zero and $m$ tends to infinity.

The following table was computed via double-precision arithmetic in FORTRAN on an Amdahl 470/V7 computer. All entries are rounded down.

Lower bounds on $\lim \sup \|P\|$

\begin{tabular}{rcrrrrrc}
\hline$k$ & $\max p(t)$ & $k$ & $\max p(t)$ & $k$ & $\max p(t)$ & $k$ & $\max p(t)$ \\
\hline 2 & 2.0000 & 7 & 7.7939 & 12 & $9.02 \times 10$ & 27 & $9.45 \times 10^{5}$ \\
3 & 2.5078 & 8 & 11.8194 & 15 & $5.13 \times 10^{2}$ & 30 & $6.60 \times 10^{6}$ \\
4 & 3.0814 & 9 & 18.7344 & 18 & $3.17 \times 10^{3}$ & 33 & $4.67 \times 10^{7}$ \\
5 & 3.9686 & 10 & 30.7986 & 21 & $2.05 \times 10^{4}$ & 36 & $3.34 \times 10^{8}$ \\
6 & 5.4087 & 11 & 52.1254 & 24 & $1.37 \times 10^{5}$ & 39 & $2.42 \times 10^{9}$ \\
\hline
\end{tabular}

This table, in which the exponential growth is clear, is associated with Theorem 2 above. A corresponding table of lower bounds on $\lim \sup \|P\|$ for the node assignment of Theorem 3 can be computed from the fact that the Lebesgue function for polynomial interpolation on the Chebyshev nodes attains its maximum 
at $t=1$; see [7, Eq. (4.2.19)]. The first few entries of such a table are:

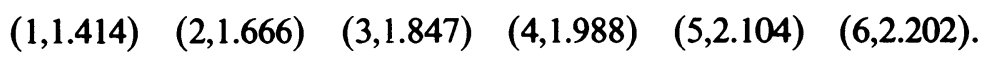

A later entry is $(35,3.243)$. The logarithmic growth is clear. For $k=1$ with arbitrary knots it can be shown that $\|P\| \leqslant \sqrt{2}$ when nodes are specified by (3.2) above. Whether the other bounds are "good" bounds for the arbitrary knot case is problematical.

4. Remarks. For one-sided $q$-splines with spline knots $t_{i}=\left(1-q^{i}\right) /(1-q)$, $i=\ldots,-1,0,1,2, \ldots$, and interpolation nodes $\tau_{i}=t_{i}+\theta q^{i}$, where $\theta$ is fixed, $0 \leqslant \theta \leqslant 1$, S. L. Lee [5] has considered eigensplines, i.e., nontrivial splines $s(t)$ satisfying $s(t)=\lambda s(1+q t)$ for some fixed eigenvalue $\lambda$. Setting $\lambda=-1$ yields, for each $k \geqslant 2$, a certain equation $F_{k}(q, \theta)=0$. If $q$ and either $\theta_{1}$ or $\theta_{2}$ defined above satisfy this equation, then two-sided $q$-spline interpolation is unbounded. Lee [5] has shown that $F_{k}(0+, \theta)=C\left[2 \theta^{k}-1\right]\left[2(1-\theta)^{k}-1\right]$.

For quadratic splines with arbitrary knots $t_{i}$, Demko [3] has shown that interpolation is bounded independently of $t_{i}$ and $\tau_{i}$ if the nodes $\tau_{i}$ satisfy $\tau_{i}=t_{i+2}-$ $\lambda_{i}\left(t_{i+2}-t_{i+1}\right)$ with $\lambda_{i}^{2} \leqslant \gamma<\frac{1}{2}$ and $\left(1-\lambda_{i}\right)^{2} \leqslant \gamma<\frac{1}{2}$. Consequently, for $k=2$, the results of Theorem 1 above with (2.5) $a$ and (2.6) $a$ are valid for all $q$ and not just as $q$ tends to zero.

Acknowledgement. I would like to thank S. D. Riemenschneider and A. Sharma for much useful advice while I was working on this problem.

Department of Mathematics

University of Pittsburgh

Pittsburgh, Pennsylvania 15260

1. C. DE BoOR, A Practical Guide to Splines, Applied Mathematical Sciences Series, Vol. 27, Springer-Verlag, New York, 1978.

2. C. DE BOOR, "On bounding spline interpolation,” J. Approx. Theory, v. 14, 1975, pp. 191-203.

3. S. Demko, "Interpolation by quadratic splines," J. Approx. Theory, v. 23, 1978, pp. 392-400.

4. H. W. Gould, Combinatorial Identities, Henry W. Gould, Morgantown, 1972.

5. S. L. LEE, private communication.

6. F. Richards, "The Lebesgue constants for cardinal spline interpolation," J. Approx. Theory, v. 14, 1975, pp. 83-92.

7. T. J. Rivin, An Introduction to the Approximation of Functions, Blaisdell, New York, 1969. 